\title{
SEMANTICAL COMPLETENESS THEOREMS IN LOGIC AND ALGEBRA
}

\author{
VLADIMIR LIFSCHITZ
}

\begin{abstract}
A few well-known algebraic theorems, including the Nullstellensatz and a form of the duality theorem of Farkas-Minkowsky, are shown to be corollaries to the completeness of the hyper-resolution method in proof theory.
\end{abstract}

Completeness theorems in logic have the form: if a formula (of a given formal language) is true (in a given semantics) then it is provable (in a given formal system). It may be observed that some important theorems in algebra have essentially the same form.

Consider, e.g., Hilbert's Nullstellensatz:

If $f$ is a polynomial of $K\left[x_{1}, \ldots, x_{n}\right]$ which vanishes at all common zeros of $f_{1}, \ldots, f_{l}$ in any extension of $K$, then $f^{\rho}=h_{1} f_{1}+\cdots+h_{l} f_{l}$ for some natural number $\rho$ and polynomials $h_{1}, \ldots, h_{l}$ of $K\left[x_{1}, \ldots, x_{n}\right]$.

Assume we are given polynomials $f, f_{1}, \ldots, f_{l}$ and want to prove that $f$ vanishes at all common zeros of $f_{1}, \ldots, f_{l}$ in any extension of $K$.

To give such a proof, we can try, for example, to represent $f$ as a linear combination of $f_{1}, \ldots, f_{l}$. If this does not work, we can try to find such a representation for $f^{\rho}$ for some natural number $\rho$; that would imply the validity of the above assertion too. Now the Nullstellensatz says that a proof of this particular form is always possible, provided the assertion to be proved is true. This remark shows the resemblance between the Nullstellensatz and completeness theorems in logic.

In this paper we show that this is not a mere superfluous analogy; on the contrary, some algebraic completeness theorems, including the Nullstellensatz, are simply corollaries to a well-known logical completeness result: the completeness of Robinson's calculus based on the hyper-resolution principle [1]. (The importance of the hyper-resolution method for establishing connections between proof theory and other parts of mathematics was first noted by Matijasevich [2].)

Connections between the Nullstellensatz and logic are also discussed by Scarpellini [3] and Whiteley [4]. Scarpellini uses a version of Gentzen's Hauptsatz rather than Robinson's theorem, which makes his analysis more complicated.

I am very much indebted to M. Gelfond, S. Maslov, Yu. Matijasevich, and G. Mints for their helpful comments.

Received by the editors June 5, 1978; presented to the Society, August 12, 1978.

AMS (MOS) subject classifications (1970). Primary 02D99.

Key words and phrases. Hyper-resolution principle, Nullstellensatz. 
1. The hyper-resolution principle. Let $T$ be a set of propositional formulas, called axioms, each of the form

$$
\mathbb{i}_{i=1}^{m} A_{i} \rightarrow \bigvee_{j=1}^{n} B_{j}
$$

where $A_{1}, \ldots, A_{m}, B_{1}, \ldots, B_{n}$ are atoms (i.e. propositional variables) and $m+n$ $>0$. A clause is a set of atoms; for any clause $\Gamma, \bar{\Gamma}$ is the disjunction of the elements of $\Gamma$ in a fixed order. Consider the following calculus $H_{T}$ : the objects derivable in $H_{T}$ are clauses, and the postulates of $H_{T}$ are

$$
\frac{\Gamma_{1} \cup\left\{A_{1}\right\}, \ldots, \Gamma_{m} \cup\left\{A_{m}\right\}}{\Gamma_{1} \cup \ldots \cup \Gamma_{m} \cup\left\{B_{1}, \ldots, B_{n}\right\}}
$$

$\left(\Gamma_{1}, \ldots, \Gamma_{m}\right.$ vary through arbitrary clauses) for every axiom (1). We write derivations in $H_{T}$ in tree form.

Example. Let $T=\{A \rightarrow B \vee C, A, \neg B\}$. Then $H_{T}$ has three postulates: the rule

$$
\frac{\Gamma \cup\{A\}}{\Gamma \cup\{B, C\}}
$$

the axiom $\{A\}$, and the rule

$$
\frac{\Gamma \cup\{B\}}{\Gamma}
$$

Robinson's theorem on the completeness of the hyper-resolution method [1] in the propositional case can be stated as follows:

ROBINSON's THEOREM, Version 1. If $T$ is contradictory then $\vdash_{H_{T}} \varnothing$.

We need the following generalization to this result.

Robinson's TheOREM, Version 2. For any clauses $\Gamma_{1}, \ldots, \Gamma_{l}, \Delta$, if $T$ 十 $\left(\bar{\Gamma}_{l}, \& \ldots \& \bar{\Gamma}_{l}\right) \rightarrow \bar{\Delta}$ then $\Gamma_{1}, \ldots, \Gamma_{l} \vdash_{H_{T}} \Delta^{\prime}$ for some $\Delta^{\prime} \subset \Delta$.

Example. For $T$ as in the example above, let $l=0, \Delta=\{C\}$. Since $C$ is derivable from $T$, the theorem asserts the derivability of $\{C\}$ or $\varnothing$ in $H_{T}$. Here is a derivation of $\{C\}$ in $H_{T}$ :

$$
\text { (2a) } \frac{\{A\}}{\{B, C\}}
$$

Proof. Let $\Delta=\left\{C_{1}, \ldots, C_{m}\right\}$. Assume that $T \vdash\left(\bar{\Gamma}_{1} \& \ldots \& \bar{\Gamma}_{l}\right) \rightarrow \bar{\Delta}$. Then

$$
T^{\prime}=T \cup\left\{\bar{\Gamma}_{1}, \ldots, \bar{\Gamma}_{l}, \neg C_{1}, \ldots, \neg C_{m}\right\}
$$

is contradictory.

$H_{T^{\prime}}$ consists of the postulates of $H_{T}$, the axioms $\Gamma_{1}, \ldots, \Gamma_{l}$, and the rules

$$
\frac{\Gamma \cup C_{i}}{\Gamma} \text {. }
$$


By Robinson's Theorem, Version 1, there exists a derivation of $\varnothing$ in $H_{T^{\prime}}$. Consider an application of (3) in this derivation. Replace every clause $\Sigma$ below the premise of this application by $\Sigma \cup\{C\}$. Every application of a rule of inference becomes an application of the same rule with one exception: (3) becomes a pair of equal clauses. Delete one of them; the resulting figure is again a derivation in $H_{T^{\prime}}$. Repeat this procedure until all applications of the form (3) are eliminated. The resulting figure is a derivation in $H_{T^{\prime}}$ of a part $\Delta^{\prime}$ of $\Delta$ without applications of (3). It can be obviously considered a derivation in $H_{T}$ of $\Delta^{\prime}$ from $\Gamma_{1}, \ldots, \Gamma_{l}$. Q.E.D.

In the applications of the hyper-resolution method in this paper we consider first order languages rather than the language of propositional calculus. For this reason, we consider one more version of Robinson's theorem.

Let $T$ be a first order theory with nonlogical axioms of the form (1). (Equality, if available, is considered a nonlogical symbol, i.e. the axioms for it are considered nonlogical.) We assume that the set of nonlogical axioms is closed under substitution: the result of substituting terms for free variables in an axiom is again an axiom (e.g., the reflexivity of equality should be stated in the form $r=r, r$ a term, rather than $x=x$ ).

Robinson's TheOrem, Version 3. For any clauses $\Gamma_{1}, \ldots, \Gamma_{l}, \Delta$, if

$$
\left(\bar{\Gamma}_{1} \& \ldots \& \bar{\Gamma}_{l}\right) \rightarrow \bar{\Delta}
$$

is valid in every model of $T$ then $\Gamma_{1}, \ldots, \Gamma_{l} \vdash_{H_{T}} \Delta^{\prime}$ for some $\Delta^{\prime} \subset \Delta$.

Proof. If (4) is valid in every model of $T$ then it is derivable in predicate calculus from the universal closures of nonlogical axioms of $T$. Both (4) and the axioms are quantifier-free, and the set of axioms is closed under substitution; it follows that (4) is derivable from nonlogical axioms in propositional calculus, and we can refer to Version 2. Q.E.D.

An application of (2) is trivial if $A_{i} \in \Gamma_{i}$ for some $i$. The conclusion of a trivial application contains one of its premises; in terms of corresponding disjunctions, a trivial application simply weakens one of the premises by adding more disjunctive members. We are going to show now that trivial applications can be always deleted from a given derivation.

LEMMA 1. Let $\Pi$ be obtained from $\Sigma_{1}, \ldots, \Sigma_{m}$ by one application of a rule of $H_{T}$. For any $\Sigma_{1}^{\prime} \subset \Sigma_{1}, \ldots, \Sigma_{m}^{\prime} \subset \Sigma_{m}$,

(a) $\Sigma_{i}^{\prime} \subset \Pi$ for some $i$, or

(b) some $\Pi^{\prime} \subset \Pi$ can be obtained from $\Sigma_{1}^{\prime}, \ldots, \Sigma_{m}^{\prime}$ by one application of the same rule.

Proof. Consider an application of (2) leading from $\Sigma_{1}, \ldots, \Sigma_{m}$ to $\Pi$; for this application,

$$
\begin{gathered}
\Sigma_{1}=\Gamma_{1} \cup\left\{A_{1}\right\}, \ldots, \quad \Sigma_{m}=\Gamma_{1} \cup\left\{A_{m}\right\}, \\
\Pi=\Gamma_{1} \cup \ldots \cup \Gamma_{m} \cup\left\{B_{1}, \ldots, B_{n}\right\} .
\end{gathered}
$$


Consider two cases.

(a) $A_{i} \notin \Sigma_{1}^{\prime}$ for some $i$. Then $\Sigma_{i}^{\prime} \subset \Gamma_{i} \subset \Pi$.

(b) $A_{i} \in \Sigma_{i}^{\prime}$ for every $i$. Then $\Sigma_{i}^{\prime}=\Gamma_{i}^{\prime} \cup\left\{A_{i}\right\}$ for $\Gamma_{i}^{\prime}$ defined by $\Gamma_{i}^{\prime}=\Sigma_{i}^{\prime} \backslash\left\{A_{i}\right\}$, and one application of (2) to $\Sigma_{1}^{\prime}, \ldots, \Sigma_{m}^{\prime}$ gives $\Pi^{\prime}=\Gamma_{1}^{\prime} \cup \ldots \cup \Gamma_{m}^{\prime} \cup$ $\left\{B_{1}, \ldots, B_{n}\right\} \subset \Pi$.

LEMMA 2. For any derivation of a clause $\Pi$ in $H_{T}$ there exists a derivation of some $\Pi^{\prime} \subset \Pi$ in $H_{T}$ without trivial applications of rules of inference.

Proof. Consider a trivial application of a rule in a derivation. The conclusion $\Pi$ of this application contains one of its premises $\Sigma$. Replace the subtree ending with $\Pi$ by the subtree ending with $\Sigma$ (i.e. delete the premises other than $\Sigma$, along with their derivations, and $\Pi$ from the given derivation). The step leading to the clause immediately below $\Sigma$ is not necessarily an application of a rule of inference; however, Lemma 1 implies that some atoms can be deleted successively from the clauses below $\Sigma$ in such a way that every step in the resulting figure will either (a) lead to the conclusion equal to one of the premises, or (b) be an application of a rule of inference. After deleting repeating clauses below $\Sigma$ in this figure we get a derivation in $H_{T}$.

Repeat this procedure as many times as necessary to get rid of all trivial applications; the process will terminate, since each time at least one clause is deleted.

Lemma 2 and Robinson's Theorem, Version 3, imply:

Robinson's TheOREM, Version 4. For any clauses $\Gamma_{1}, \ldots, \Gamma_{l}, \Delta$, if (4) is valid in every model of $T$ then there exists a derivation of some $\Delta^{\prime} \subset \Delta$ from $\Gamma_{1}, \ldots, \Gamma_{l}$ in $H_{T}$, containing no trivial applications of rules of inference.

2. Linear equations and inequalities. For a given field $K$, consider the first order language with the following nonlogical symbols:

for any $\alpha \in K$, a unary function symbol for multiplication by $\alpha$,

the binary function symbol + ,

the unary predicate symbol $=0$.

Terms of this language are essentially linear forms over $K$, and atomic formulas are homogeneous linear equations. For any terms $r, s, r=s$ stands for $r-s=0$.

Consider the theory in this language with the following nonlogical axioms:

$$
\begin{gathered}
0 \cdot r=0, \\
r_{1}=0 \rightarrow r_{2}=0 \quad\left(r_{1}, r_{2} \text { represent the same linear form }\right), \\
(r=0 \& s=0) \rightarrow r+s=0, \\
r=0 \rightarrow \alpha r=0 .
\end{gathered}
$$

These axioms imply all equality axioms and all axioms of linear spaces over $K$. It follows that the models of this theory factorized w.r.t. $x=y$ are precisely the linear spaces over $K$.

$H_{T}$ consists of the axiom

$$
\{0 \cdot r=0\}
$$


and the rules of inference

$$
\frac{\Gamma \cup\left\{r_{1}=0\right\}}{\Gamma \cup\left\{r_{2}=0\right\}}, \quad r_{1}, r_{2},
$$

represent the same linear form,

$$
\begin{gathered}
\frac{\Gamma \cup\{r=0\}, \Delta \cup\{s=0\}}{\Gamma \cup \Delta \cup\{r+s=0\}}, \\
\frac{\Gamma \cup\{r=0\}}{\Gamma \cup\{\alpha r=0\}} .
\end{gathered}
$$

Let each of the clauses $\Gamma_{1}, \ldots, \Gamma_{l}$ be a singleton: $\Gamma_{i}=\left\{r_{i}=0\right\}$. A derivation in $H_{T}$ from $\Gamma_{1}, \ldots, \Gamma_{l}$ without trivial applications of the rules of inference consists of singletons; moreover, for each member $\{s=0\}$ of this derivation $s$ is obviously a linear combination of $r_{1}, \ldots, r_{l}$. Hence Robinson's Theorem expresses in this case the following well-known fact of linear algebra:

If every solution of simultaneous homogeneous linear equations $r_{1}=0, \ldots, r_{l}=0$ is a solution of a homogeneous linear equation $s=0$ then $s$ is a linear combination of $r_{1}, \ldots, r_{l}$.

By "solution" we understand here a solution in any linear space over $K$. Considering solutions in $K$ itself is known to be sufficient; this refinement is not directly implied, however, by Robinson's theorem.

By changing some details in the above construction, we arrive at a similar result for linear inequalities. Let $K$ be an ordered field, and the only predicate symbol in the language be $\geqslant 0$ instead of $=0 ; r=s$ stands now for $r-s>0 \& s-r \geqslant 0$. Replace $=0$ in the nonlogical axioms by $>0$ and add the restriction $\alpha>0$ to (8). The models of this theory factorized w.r.t. $x=y$ are precisely the partially ordered linear spaces over $K$.

Repeating the above argument for this theory, we get the following form of the duality theorem of Farkas-Minkowski:

If every solution of simultaneous homogeneous linear inequalities $r_{1}>0, \ldots, r_{l}>$ 0 is a solution of a homogeneous linear inequality $s \geq 0$ then $s$ is a nonnegative linear combination of $r_{1}, \ldots, r_{l}$.

Here again the solutions in $K$ itself can be considered instead of those in linear spaces over $K$. This refinement is, in a manner of speaking, the algebraic part of the duality theorem, the rest being a proof-theoretic result.

3. The Nullstellensatz. For an arbitrary field $K$, consider now the first order theory with constants for the elements of $K$, with the binary function symbols + and $\cdot$, with the unary predicate symbol $=0$ (so that the terms and atomic formulas are polynomials and, respectively, algebraic equations over $K$ ), and with the following nonlogical axioms:

$$
\begin{gathered}
0=0, \\
\neg \alpha=0 \quad(\alpha \in K \backslash\{0\}), \\
r_{1}=0 \rightarrow r_{2}=0 \quad\left(r_{1}, r_{2} \text { are equal polynomials }\right),
\end{gathered}
$$




$$
\begin{gathered}
(r=0 \& s=0) \rightarrow r+s=0, \\
r=0 \rightarrow r s=0, \\
r s=0 \rightarrow(r=0 \vee s=0) .
\end{gathered}
$$

Define $r=s$ as in the case of linear equations above; then one can prove the equality axioms, the axioms of integral domain, and the diagram of $K$. It follows that the models of the theory in question, factorized w.r.t. $x=y$, are the integral domains containing $K$.

$H_{T}$ consists of the axiom

$$
\{0=0\}
$$

and the rules of inference

$$
\begin{gathered}
\frac{\Gamma \cup\{\alpha=0\}}{\Gamma} \quad(\alpha \in K \backslash\{0\}), \\
\frac{\Gamma \cup\left\{r_{1}=0\right\}}{\Gamma \cup\left\{r_{2}=0\right\}} \quad\left(r_{1}, r_{2}\right. \text { are equal polynomials), } \\
\frac{\Gamma \cup\{r=0\}, \Delta \cup\{s=0\}}{\Gamma \cup \Delta \cup\{r+s=0\}} \\
\frac{\Gamma \cup\{r=0\}}{\Gamma \cup\{r s=0\}} \\
\frac{\Gamma \cup\{r s=0\}}{\Gamma \cup\{r=0, s=0\}} .
\end{gathered}
$$

As in the two cases above, we want to describe the structure of the clauses derivable in $H_{T}$ from a set of singletons. The situation here is more complicated, since, because of the rule $\left(14^{\prime}\right)$, such clauses are not necessarily singletons (even if trivial applications are forbidden). We get over this difficulty by showing that applications of rules of inference in a given derivation can be always rearranged in such a way that (14') will be applied at the very bottom of the derivation only, after all other rules of inference.

Assume, as a matter of fact, that there is an application (we call it signed) of one of the rules $\left(10^{\prime}\right)-\left(13^{\prime}\right)$ immediately below an application of $\left(14^{\prime}\right)$; if the signed rule is (12), assume, for definiteness, that (14') is above its left premise. If both $r=0$ and $s=0$ in the conclusion of the signed application are different from $A_{1}$ explicitly shown in the scheme (2) of the signed application then (14') and the signed application can be permuted in the following way:

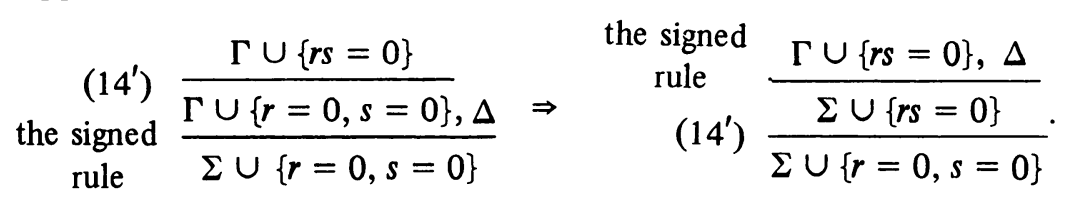

Here $\Delta$ is the second premise of the signed application, if it is an application of (12); otherwise it should be dropped.

Let now at least one of $r=0, s=0$ be $A_{1}$ of the signed application; for definiteness, let $A_{1}$ be $r=0$. If the signed application is trivial, delete it altogether, 
as in the proof of Lemma 2. If not, then $(r=0) \notin \Gamma \cup\{s=0\}$. Depending on whether the signed rule is $\left(10^{\prime}\right),\left(11^{\prime}\right),\left(12^{\prime}\right)$ or $\left(13^{\prime}\right)$, change the derivation according to one of the schemata below.
$\left(14^{\prime}\right) \frac{\Gamma \cup\{\alpha s=0\}}{\Gamma \cup\{\alpha=0, s=0\}}$
$\left(10^{\prime}\right) \frac{\Gamma \cup\{s=0\}}{\Gamma \cup}$$\Rightarrow$
$\left(13^{\prime}\right) \frac{\Gamma \cup\{\alpha s=0\}}{\Gamma \cup\left\{\alpha^{-1} \alpha s=0\right\}}$,
(14') $\frac{\Gamma \cup\left\{r_{1} s=0\right\}}{\Gamma \cup\left\{r_{1}=0, s=0\right\}}$
$\left(11^{\prime}\right) \frac{\Gamma \cup\left\{r_{2}=0, s=0\right\}}{\Gamma}$$\Rightarrow$
$\left(11^{\prime}\right) \frac{\Gamma \cup\left\{r_{1} s=0\right\}}{\Gamma \cup\left\{r_{2} s=0\right\}}$,

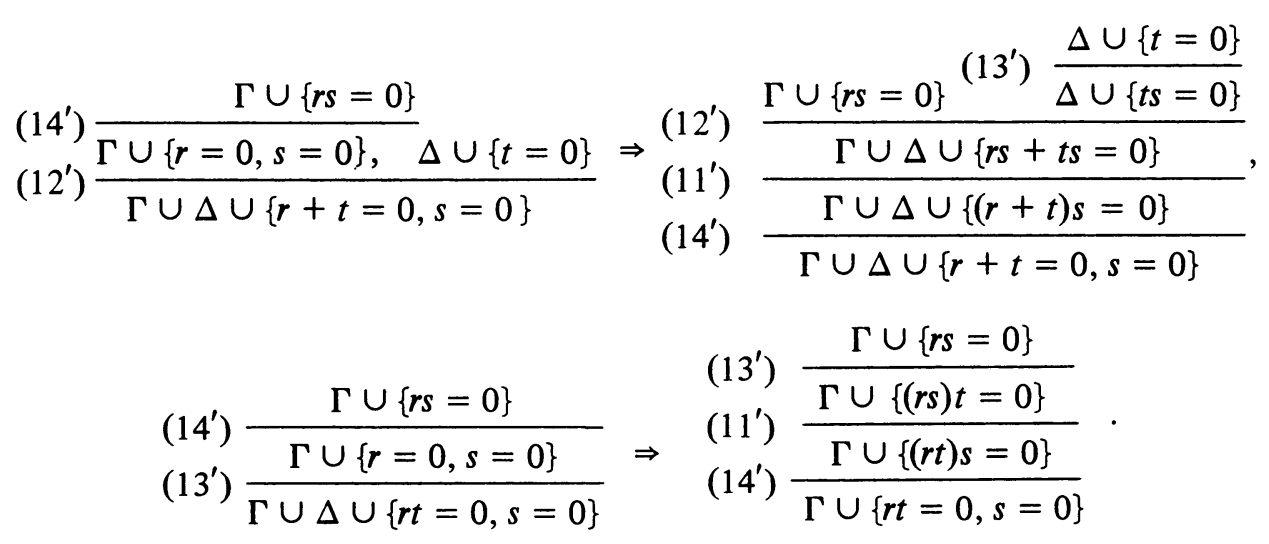

By a series of applications of this procedure we construct a derivation with (14') applied after all other rules only. This property will still hold after we eliminate all trivial applications of rules of inference as in the proof of Lemma 2.

Assume now that every solution of $r_{1}=0, \ldots, r_{l}=0$ is a solution of $s=0$. By Robinson's theorem, a part $\Delta^{\prime}$ of $\Delta=\{s=0\}$ (i.e. $\varnothing$ or $\Delta$ ) can be derived from $\Gamma_{1}=\left\{r_{1}=0\right\}, \ldots, \Gamma_{l}=\left\{r_{l}=0\right\}$ without trivial applications of rules of inference. Consider a derivation of $\Delta^{\prime}$ from $\Gamma_{1}, \ldots, \Gamma_{l}$ which contains applications of $\left(14^{\prime}\right)$ at the very end only. There is a clause $\Sigma$ in the derivation separating the applications of $\left(14^{\prime}\right)$ from applications of the other rules. Since $\Sigma$ is derived from singletons using $\left(10^{\prime}\right)-\left(13^{\prime}\right)$ only, it contains at most one element.

Case 1. $\Sigma$ is a singleton. Since $\Delta^{\prime}$ is derived from $\Sigma$ by a series of applications of (14'), it is nonempty; hence $\Delta^{\prime}=\Delta=\{s=0\}$. Furthermore, $\{s=0\}$ can be only derived from $\Sigma$ by a series of applications of $\left(14^{\prime}\right)$ if $\Sigma=\left\{s^{\rho}=0\right\}$ for some natural number $\rho$. On the other hand, since $\Sigma$ is nonempty, it is derived from $\Gamma_{1}, \ldots, \Gamma_{l}$ without $\left(10^{\prime}\right)$; hence all clauses above $\Sigma$ are singletons and, obviously, linear combinations of $r_{1}=0, \ldots, r_{l}=0$. It follows that $s^{\rho}$ is a linear combination of $r_{1}, \ldots, r_{l}$.

Case 2. $\Sigma=\varnothing$. Then $\Sigma$ coincides with $\Delta^{\prime}$, so that $\Delta^{\prime}=\varnothing$ and $\left(14^{\prime}\right)$ is not applied at all. The rule applied at the very end is then $\left(10^{\prime}\right)$ and this is the only application of $\left(10^{\prime}\right)$. Let its premise be $\{\alpha=0\}$; then $\alpha$ is a linear combination of $r_{1}, \ldots, r_{l}$. It follows that $s^{0}=1$ is also a linear combination of $r_{1}, \ldots, r_{l}$. 
Thus we proved the Nullstellensatz. Here again, as in the case of linear equations and inequalities, we consider zeros in the structures more general than in the traditional form of the theorem: in integral domains instead of fields. However, in this case the difference is obviously inessential, since any integral domain is imbeddable into a field.

It should be noted that the additional argument concerning permutability of inferences that we needed to derive the Nullstellensatz from Robinson's theorem is proof-theoretic, not algebraic in nature; cf. [5]. The only algebraic facts we had to use were immediate consequences of the definition of field. It is fair to say that the Nullstellensatz is a purely proof-theoretic result.

\section{REFERENCES}

1. J. A. Robinson, Automatic deduction with hyper-resolution, Internat. J. Comput. Math. 1 (1965), 227-234.

2. Yu. V. Matijasevich, On metamathematical approach to proving theorems of discrete mathematics, Seminars in Mathematics, vol. 49, Steklov Mathematical Institute, Leningrad, 1975, pp. 31-50. (Russian, with English summary)

3. B. Scarpellini, On the metamathematics of rings and integral domains, Trans. Amer. Math. Soc. 138 (1969), 71-76.

4. W. Whiteley, Logic and invariant theory, Ph.D. Thesis, M.I.T., Cambridge, Mass., 1971.

5. S. C. Kleene, Permutability of inferences in Gentzen's Calculi LK and LJ, Mem. Amer. Math. Society, no. 10, 1952.

Department of Mathematics, Brigham Young University, Provo, Utah 84602

Current address: Department of Mathematical Sciences, University of Texas at El Paso, El Paso, Texas 79968 